\title{
SAR Study of $\beta$-Aminoacyl-Containing Cyclic Hydrazide Derivatives as DPP-IV Inhibitors
}

\author{
Mi Ae Jun, Mi Sik Shin, Woul Seong Park,' Seung Kyu Kang, Ki Young Kim, Sang Dal Rhee, \\ Duck Hyung Lee, 'Hyae Gyeong Cheon, Jin Hee Alın, and Sung Soo Kim ${ }^{*}$ \\ Ding Discovery Division, Korea Research Instifute of Chemical Technolog: Daejeon 305-600, Korea \\ E-mal: hahnakictrek \\ ${ }^{\dagger}$ Department of Chemistry, Sogang University, Seoul 121-742, Korea \\ Received August 3, 2008
}

\begin{abstract}
In continuation of our efforts to further derivatize dipeptidyl peptidase IV (DPP-IV) inhibitors, a series of $\beta$ aninoacyl-containing 5-, 6- and 7- membered cyclic hydrazide derivatives was synthesized. All the compounds were evaluated for their ability to inhibit DPP-IV, and an optinum structural unit on basic skeleton is identified to show good in vitro activity.
\end{abstract}

Key Words : Dipeptidyl peptidase IV, Diabetes, Cyclic hydrazide

\section{Introduction}

A non-insulin dependent diabetes mellitus (NIDDM) is characterized by chronic hyperglycemia, and belongs to a group of metabolic disorders with multiple etiologies. It is very common and may result from insulin resistance, inadequate secretion of insulin, hepatic glucose overproduction, or glucose intolerance. ${ }^{1}$

GLP- ${ }^{2}$ is released from $L$ cells of the small intestine in response to digestion of food, and plays an important role in secretion of insulin. Increased activity of GLP-1 will lead to sustained insulin secretion, which normalize an elevated glucose level. It also retards gastric emptying, induction of satiety and stimulation, regeneration \& differentiation of islet $\beta$-cells. ' A dipeptidyl peptidase IV (DPP-IV), a serine protease present in many tissues, and body fluids exist either with membrane bound or soluble enzyme. It degrades GLP1 (GLP-1[7-36]amide) into inactive GLP[9-36]amide ${ }^{4.5}$ at $N$-terminus position. Inhibition of DPP-IV increases the concentration of GLP-1 as a result increases insulin secretion, ${ }^{6}$ which can ameliorate hyperglycemia in type 2 diabetes. In recent past, several reports on use of small molecules as inhibitors of DPP-IV is available in literauture. $^{?}$

In our previous paper, ${ }^{8}$ we have described the synthesis and biological evaluation of $\beta$-aminoacyl-containing cyclic hydrazine derivatives with only 6 examples. In continuation of our efforts, we have further derivatized the core compounds with diversified substituents, in order to find a potential candidate as DPP-IV inhibitor. We now wish to

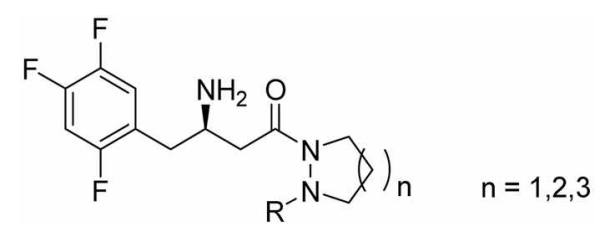

Figure 1. $\not$-aminoacyl-containing cyclic hydrazide derivatives. report here the detalled $\mathrm{SAR}$ study of $\beta$-aminoacyl-containing cyclic hydrazide derivatives as DPP-IV inhibitors.

A series of $\beta$-aminoacyl-containing cyclic hydrazine derivatives was synthesized by using the route shown in Scheme 1. The detailed synthetic explanation was described in our previous publication. ${ }^{8}$

5-, 6- and 7-Membered cyclic hydrazide derivatives with $\beta$-aminoacyl group were evaluated in vino for their inhibitions against DPP-IV. MK-0431 was used as a reference compound. Compounds which showed more than $50 \%$ inhibition of DPP-IV at $100 \mathrm{nM}$, were considered as promising and the $\mathrm{IC}_{5 i}$ values of the compounds were determined. The data are compared with ring size and also various functionalities such as acyl, benzoyl, urea, sulfonyl, carbamate, and alkyl groups. Basic compounds ( $\mathrm{R}=\mathrm{H}, \mathbf{5 - 1}$, 6-1 and 7-1) couldn't reach 50\% inhibition at $100 \mathrm{nM}$, however benzoyl substituents promoted activity. More particularly 6- and 7-membered benzoyl hydrazides (6-2 and 72) showed good in vitro inhibitory activities with $\mathrm{IC}_{50}$ values

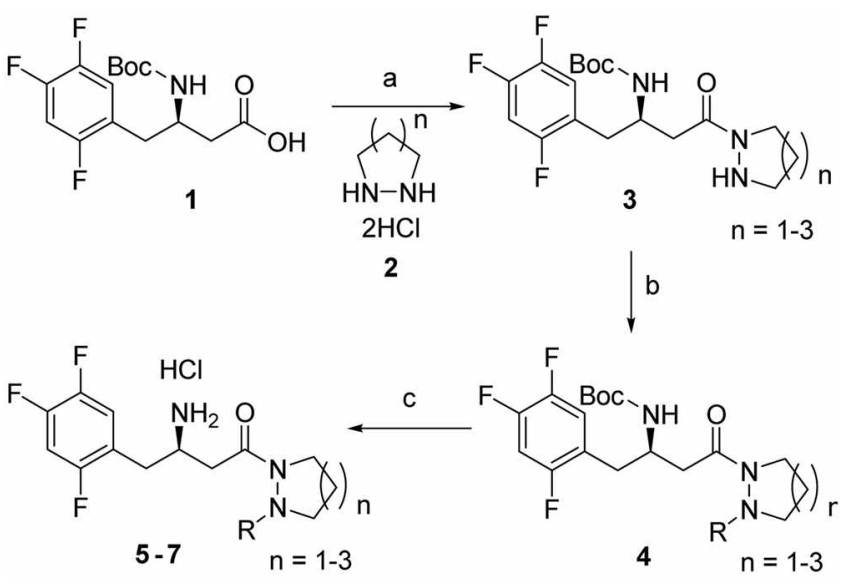

Scheme 1. Reagents and conditions: (a) compound 2, triethylamine. EDCI, $\mathrm{CH}_{2} \mathrm{Cl}_{2}$, room temperature: (b) electrophiles, $\mathrm{CH}_{2} \mathrm{Cl}_{3}$, triethylanine, room temperature; (c) $\mathrm{HCl}$. dioxane, room temperature. 
Table 1. Inhibitory activity of $\beta$-aminoacyl-containing cyclic hydrazide derivatives against DPP-IV<smiles>[R]N1CCCN1C(=O)C[C@@H](N)Cc1cc(F)c(F)cc1F</smiles>

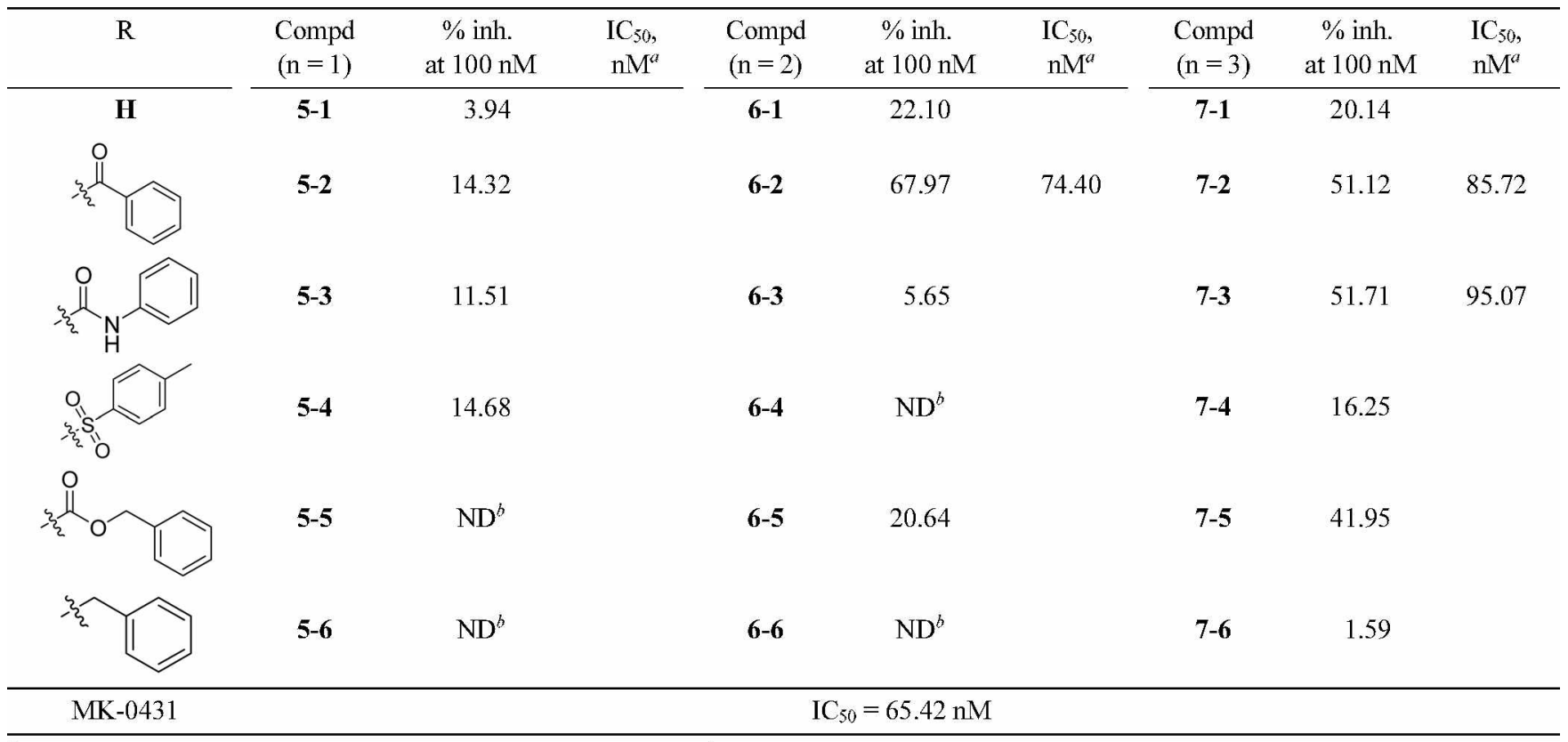

${ }^{a} \mathrm{IC}_{50}$ values were determined from direct regression curve analysis. ${ }^{b}$ not determined.

of $74.40 \mathrm{nM}$ and $85.72 \mathrm{nM}$ respectively. In case of urea, 7membered hydrazide displayed a good activity with 95.07 nM. All other substituents such as sulfonyl, carbamate and aralkyl groups showed weak activities.

The benzoyl derivatives (6-2 and 7-2) being demonstrated good activity further derivatized with various substituted benzoyl derivatives and evaluated. Some compounds (7-11. $7-13,7-14,7-15,7-17,7-18$ and 7-22) showed good activities and compound (7-18) is found to be most active with an $\mathrm{IC}_{50}$ value $32.80 \mathrm{nM}$. The details are tabulated in Table 2.

Lrea based substituent also being active, it is further derivatized with various substituents and evaluated. Compound 7-39 showed better activity than other urea based derivatives, and the details of activity data is tabulated in Table 3.

From the SAR data, we have chosen compound 7-18 to evaluate in vivo for their ability to reduce DPP-IV activity in normal $\mathrm{C} 57 \mathrm{BL} / 6 \mathrm{~J}$ mice. Oral administration of compounds $\mathbf{7 - 1 8}$, at $10 \mathrm{mg} / \mathrm{kg}$ dose, resulted in ca $70 \%$ inhibition of plasma DPP-IV activity after $2 \mathrm{~h}$.

\section{Conclusion}

Diverse $\beta$-aminoacyl-containing 5-, 6- and 7-membered cyclic hydrazide derivatives were synthesized and evaluated for their ability to inhibit dipeptidyl peptidase IV (DPP-IV). Among them, 7-18 emerged as the most active compound with an $\mathrm{IC}_{50}$ value of $32.8 \mathrm{nM}$, and evaluated for its in vino DPP-IV inhibitory activity.

\section{Experimental}

General. All reported yields are isolated yields after column chromatography or crystallization. ${ }^{\text {H }} \mathrm{H}-\mathrm{NMR}$ spectra were obtained on FT- $\mathrm{MR}$ Varian GEMNI-200FT or Bruker AVANCE-300 with TMS as internal reference. MS spectra were obtained on a Shimadzu QP5050 spectrograph.

Synthetic Procedure for Representative Compound 7 18: A mixture of (R)-tert-butyl 4-(1,2-diazepan-1-yl)-4-oxo1-(2,4,5-trifluorophenyl)butan-2-ylcarbamate (30 mg, 0.072 mmol), Benzo[1,3]dioxole-5-carbonyl chloride (20 $\mathrm{mg}$, $0.108 \mathrm{mmol})$, and triethylamine (20 $\mu \mathrm{L}, 0.144 \mathrm{mmol})$ in $\mathrm{CH}_{2} \mathrm{Cl}_{2}(2 \mathrm{~mL})$ was stirred for $1 \mathrm{~h}$ at room temperature. The reaction mixture was diluted with brine and $\mathrm{CH}_{2} \mathrm{Cl}_{2}$. The organic layer was separated, dried and evaporated. The residue was purified by silica gel column chromatography to give (R)-tert-butyl 4-(2-(benzo[d][1,3]dioxole-5-carbonyl)1,2-diazepan-1-yl)-4-oxo-1-(2,4,5-trifluorophenyl)butan-2ylcarbamate ( $36 \mathrm{mg}, 89 \%$ ) as an oil.

${ }^{l} \mathrm{H} \mathrm{NMR}\left(\mathrm{CDCl}_{3}, 300 \mathrm{MHz}\right) \delta 7.00-6.77(\mathrm{~m}, 5 \mathrm{H}), 6.02(\mathrm{~s}$, $2 \mathrm{H}), 5.65-5.10$ (br., s, $1 \mathrm{H}), 4.22-4.09(\mathrm{~m}, 1 \mathrm{H}), 3.22-2.48$ (m, $7 \mathrm{H}), 1.91-1.42(\mathrm{~m}, 7 \mathrm{H}), 1.35(\mathrm{~s}, 9 \mathrm{H}) ;$ MS m/z $563\left(\mathrm{M}^{+}\right)$.

To a solution of (R)-tert-butyl 4-(2-(benzo[d][1,3]dioxole5-carbonyl)-1,2-diazepan-1-yl)-4-oxo-1-(2,4,5-trifluorophenyl)butan-2-ylcarbamate (50 $\mathrm{mg}, 0.089 \mathrm{mmol}$ ) in EtOAc (2 $\mathrm{mL})$, was added $4 \mathrm{M}-\mathrm{HCl} / 1,4$-dioxane $(0.5 \mathrm{~mL})$ and the mixture was stirred for $12 \mathrm{~h}$ at room temperature. The solvents were evaporated, and the residue was crystallized with ether to give (R)-3-amino-1-(2-(benzo[d] $[1,3]$ dioxole- 
Table 2. Inhibitory activity of $\beta$-aminoacyl-containing cyclic hydrazide derivatives with $\hat{N}$-acyl substituents against DPP-IV

\begin{tabular}{|c|c|c|c|c|c|c|c|c|c|}
\hline $\mathrm{R}$ & $\begin{array}{l}\text { Compd } \\
(11=1)\end{array}$ & $\begin{array}{c}\mathrm{n}=1 \\
\% \text { inh. } \\
\text { at } 100 \mathrm{nM}\end{array}$ & $\begin{array}{l}\mathrm{IC}_{\sin } \\
\mathrm{nM^{ \prime \prime }}\end{array}$ & $\begin{array}{l}\text { Compd } \\
(11=2)\end{array}$ & $\begin{array}{c}\mathrm{n}=2 \\
\% \mathrm{inh} . \\
\text { at } 100 \mathrm{nM}\end{array}$ & $\begin{array}{l}\mathrm{IC} s \phi_{m} \\
\mathrm{nM}^{*}\end{array}$ & $\begin{array}{l}\text { Compd } \\
(n=3)\end{array}$ & $\begin{array}{c}\mathrm{n}=3 \\
\% \text { inhl. } \\
\text { at } 100 \mathrm{nM}\end{array}$ & $\begin{array}{l}\mathrm{IC}_{\text {S }} \\
\mathrm{LM}^{*}\end{array}$ \\
\hline & $5-2$ & 14.32 & & $6-2$ & 67.97 & 74.40 & $7-2$ & 51.12 & 85.72 \\
\hline & $5-7$ & ND & & $6-7$ & 2.21 & & $7-7$ & 36.82 & \\
\hline & $5-8$ & ND & & $6-8$ & 2.11 & & $7-8$ & 14.75 & \\
\hline & $5-9$ & ND & & $6-9$ & 4.47 & & $7-9$ & 28.87 & \\
\hline & $5-10$ & $\mathrm{ND}$ & & $6-10$ & 23.74 & & $7-10$ & 33.83 & \\
\hline & $5-11$ & ND & & $6-11$ & 15.46 & & $7-11$ & 55.41 & 85.04 \\
\hline & $5-12$ & ND & & $6-12$ & 0.76 & & $7-12$ & 9.54 & \\
\hline & $5-13$ & 15.32 & & $6-13$ & 34.53 & & $7-13$ & 75.08 & 35.42 \\
\hline & $5-14$ & ND & & $6-14$ & 35.15 & & $7-14$ & 55.68 & 83.07 \\
\hline & $5-15$ & ND & & $6-15$ & 35.14 & & $7-15$ & 57.14 & 82.10 \\
\hline & $5-16$ & ND & & $6-16$ & 8.10 & & $7-16$ & 2.36 & \\
\hline & $5-17$ & $\mathrm{ND}$ & & $6-17$ & 38.87 & & $7-17$ & 68.42 & 41.04 \\
\hline & $5-18$ & 16.08 & & $6-18$ & 27.53 & & $7-18$ & 74.07 & 32.80 \\
\hline
\end{tabular}


Table 2. Continued

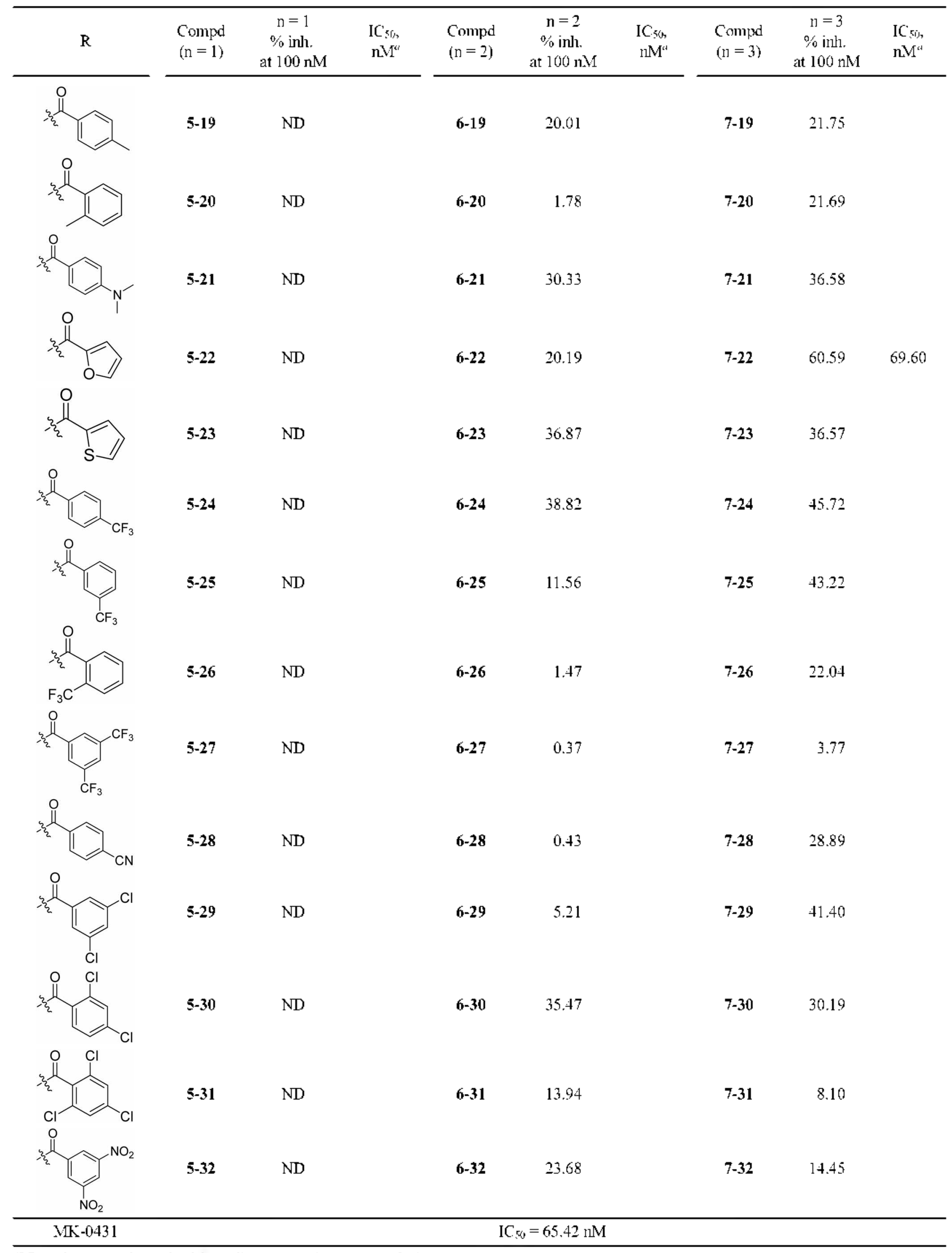


Table 3. Inhibitory activity of $B$-aminoacyl-containing cyclic hydrazide derivatives with urea substituents against DPP-IV<smiles>[R]N1CCCN1C(=O)C[C@@H](N)Cc1cc(F)c(F)cc1F</smiles>

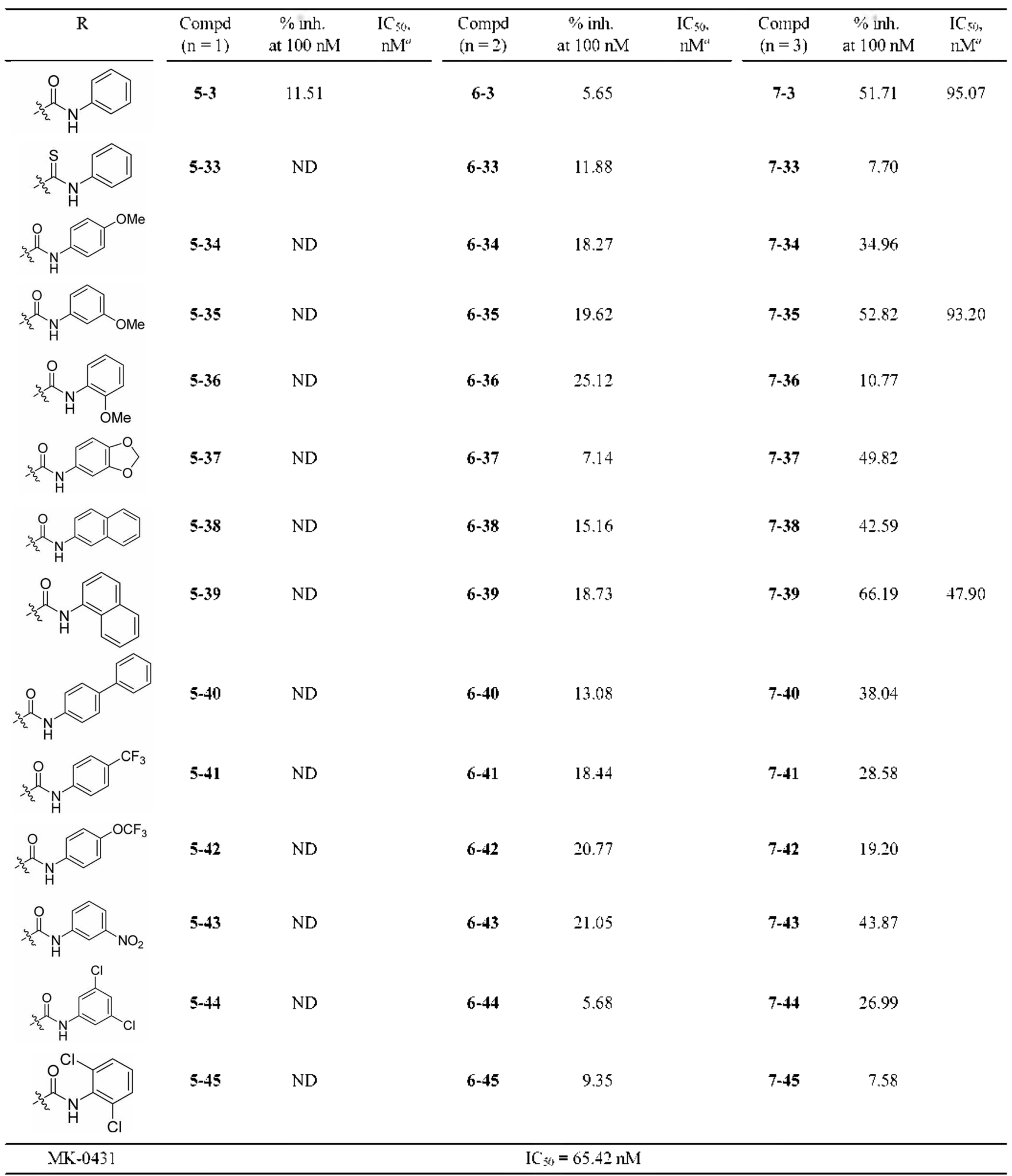

"IC $C_{s 0}$ values were determined from direct regression curve analysis. 
5-carbonyl)-1,2-diazepan-1-yl)-4-(2,4,5-trifluorophenyl)butan-1-one hydrochloride ( $40 \mathrm{mg}, 90 \%$ ) as a solid.

${ }^{l} \mathrm{H}$ NMR (DMSO-d $\left.6.500 \mathrm{MHz}\right) \delta 8.23$ (s, $1 \mathrm{H}$ ), 8.14 (s, $1 \mathrm{H}), 7.57-7.51(\mathrm{~m}, 2 \mathrm{H}), 7.13-7.10(\mathrm{~m}, 1 \mathrm{H}), 7.04-7.03(\mathrm{~m}$. $1 \mathrm{H}), 6.98-6.77(\mathrm{~m}, 1 \mathrm{H}), 6.12(\mathrm{~s}, 2 \mathrm{H}), 4.00-3.90(\mathrm{~m}, 1 \mathrm{H})$, $3.88-3.86(\mathrm{~m}, 1 \mathrm{H}), 3.75-3.72(\mathrm{~m}, 2 \mathrm{H}), 3.17-3.07(\mathrm{~m}, 1 \mathrm{H})$, $3.03-2.88(\mathrm{~m}, 3 \mathrm{H}), 2.81-2.73(\mathrm{~m}, 1 \mathrm{H}), 1.78-1.47(\mathrm{~m}, 6 \mathrm{H})$.

Determination of Inhibitory Activity against DPP-IV. $10 \mu \mathrm{L}$ of Caco-2 cell lysate was suspended in Tris-HCl (pH 7.5), and then $40 \mu \mathrm{M}$ Ala-Pro-AFC (ICN Biomedicals, Inc) was added. After treatment of compounds, the mixture was incubated for $60 \mathrm{~min}$ at $24^{\circ} \mathrm{C}$. AFC as a indicator of DPP-IV activity was detected at $405 / 510 \mathrm{~nm}(\mathrm{Ex} / \mathrm{Em})$ by Fluorometer, Synergy HT (Biotek). $\mathrm{IC}_{5 i}$ was calculated by Prism 4.0 software (GarphPad Software, Inc).

Acknowledgments. This research was supported by the Center for Biological Modulators of the 21st Century Frontier R\&D Program, Ministry of Education, Science and Technology, Korea.

\section{Reference and Notes}

1. Wild, S.; Roglic, G.; Green, A.; Sicree, R.; King, H. Diabetes Care 2004, $27,1047$.

2. (a) Kundsen, L. B. J. Med. Chem 2004, 47, 4128. (b) Drucker, D. J. Endocrinologn: 2001, 142,521.

3. (a) Holst, J. J.; Deacon, E. F. Cum Opin. Phanntacol. 2004, 4, 589. (b) Drucker, D. T. Gastroenferologt 2002, 122, 531
4. (a) Kieffer, T. J.; McIntosh, C. H. S.; Pederson, T. A. Endocrinolog. 1995, 136, 3585. (b) Deacon, C. F.; Nauck, M. A.; ToftNielson, M.; Pridal, L.; Willms, B.; Holst, J. J. Diaberes 1995, 44, 1126.

5. Mentlein, R. Regulatory Pept. 1999, 85, 9.

6. (a) Ahren, B.; Holst, J. J.; Martensson, H.; Balkan, B. Eut $J$. Phamucol. 2000, 404, 239. (b) Deacon, C. F.; Hughes, T. E.; Joist, J. J. Diabetes 1998, 47, 764. (c) Pospisilik, J. A.; Stafford, S. G.; Demuth, H.-L.; Brownsey, R.; Parkhous, W.; Finegood, D. T.; McIntosh, D. H; Pederson, R. A. Diabetes 2002, $51,943$.

7. (a) Thomberry, N. A.; Weber, A. E. Current Topics in Mediciant Chemistry 2007, 7, 557. (b) ldris, I.; Donnelly, R. Diabetes. Obesity and Metabolisn 2007, 9, 153. (c) Kim, D.; Kowalchick, I. E.; Brockunier, L. L.; Parmee, E. R.; Eiermann, G. J.; Fisher, M. H.; He, H.; Leiting, B.; Lyons, K.; Scapin, G; Pater, S. B.; Petrov, A.; Pryor, K. D.; Roy, R. S.; Wu, J. K.; Zhang, X.; Wyvratt, M. J.; Zhang, B. B.; Zhu, L.; Thonberry, N. A.; Weber, A. E. J. Med. Chem. 2008, 51, 589. (d) Kondon, T.; Nekado, T.; Sugimoto, I.; Ochi, K.; Takai, S.; Kinoshita, A.; Hatayama, A.; Yamamoto, S.; Kawabata, K.; Nakai, H.; Toda, M. Bioorg. Med. Chem. 2008, 16, 190. (e) Kondo, T.; Nekado, T.; Sugimoto, I.; Ochi, K.; Takai, S.; Kinnoshita, A.: Hatayama, A.; Yamamoto, S.; Kishikawa, K.; Nakai, H.; Toda, M. Bioorg. Med. Chem. 2008, 16, 1613. (f) Wallace, M. B.; Feng, J.; Zhang, Z.; Skene, R. J.; Shi, L.; Caster, C. L.; Kassel, D. B.; Xu, R.; Gwaltney, S. L. Bioong. Med. Chen. Lett. 2008, 18, 2362. (g) Edmondson, S. D.; Wei, L.; Xu, I.; Shang, I.; Xu, S.; Pang, J.; Chaudhary, A.; Dean, D. C.; He, H.; Leiting, B.; Lyons, K. A.; Patel, R. A.; Patel, S. B.; Scapin, G.; Wu, J. K.; Beconi, M. G; Thomberry, N. A.; Weber, A. E. Bioorg. lifed. Chem. 2008, 18, 2409.

8. Ahn, J. H.; Shin, M. S.; Jung, S. H.; Kang, S. K.; Kim, K. R.; Rhee, S. D.; Kang, N. S.; Kim, S. Y.; Sohn, S. K.; Kim, S. G.; Jin, M. S.; Lee, I. O.; Cheon, H. G.; Kim, S. S. Bioorg. Med. Chem. Lett. 2007, 17, 2622. 